\title{
Scalars with higher derivatives in supergravity and cosmology
}

\author{
Jean-Luc Lehners* \\ Max-Planck-Institute for Gravitational Physics (Albert-Einstein-Institute) \\ Am Mühlenberg 1, Potsdam D-14476, Germany \\ E-mail: jlehners@aei.mpg.de
}

We construct $\mathrm{N}=1$ supergravity extensions of scalar field theories with higher-derivative kinetic terms. Special attention is paid to the auxiliary fields, whose elimination leads not only to corrections to the kinetic terms, but to new expressions for the potential energy as well. Our formalism allows one to write a supergravity extension of any higher-derivative scalar field theory and, therefore, has applications to both particle physics and cosmological model building. As an illustration, we couple the higher-derivative DBI action to $\mathrm{N}=1$ supergravity. This displays a number of new features-including the fact that, in the regime where the higher-derivative kinetic terms become important, the potential is everywhere negative. Thus, DBI inflation cannot occur in its most interesting, relativistic regime. We show how to overcome this problem by coupling the model to an additional chiral supermultiplet. In this way, one obtains effective single real scalar field DBI models with arbitrary positive potentials. A further applications of our formalism is a supergravity versions of the ghost condensate. We discuss its physical properties, including the fact that the ghost condensate allows one to break local supersymmetry while the gravitino remains massless.

Proceedings of the Corfu Summer Institute 2012 "School and Workshops on Elementary Particle Physics and Gravity"

September 8-27, 2012

Corfu, Greece

\footnotetext{
* Speaker.
} 


\section{Introduction and motivation}

In cosmology, there are situations of interest where higher-derivative kinetic terms are important. The best-known examples are probably the models of DBI inflation [1] and k-inflation [2], where higher-derivative kinetic terms allow for slow-roll inflationary dynamics in potentials that would otherwise be too steep for successful inflation to take place. These models were inspired by certain higher-derivative terms that naturally appear in string theory, e.g. in the effective description of D-brane dynamics. An open question is whether one can derive such models from an explicit string compactification. In the present work, we will rather work from the opposite end, and ask whether these models can be formulated in $\mathscr{N}=1$ supergravity. Eventually, our approach should of course meet with the compactification approach.

Perhaps surprisingly, further motivation for studying higher-derivative cosmological models comes from the framework of eternal inflation [3]. As is well-known, eternal inflation is plagued by the measure problem, namely the necessity to choose a measure to regulate the infinities inherent to eternal inflation. However, recently a generic feature has emerged, which is of crucial importance for predictions stemming from eternal inflation: the currently known measures that do not lead to blatant paradoxes have in common that they predict that the dominant vacuum in the multiverse is the longest-lived vacuum. This so-called master vacuum has been conjectured to exhibit two important, and related, properties [4]: 1. To be very long-lived, it must be nearly supersymmetric, and hence likely has a small cosmological constant. 2. If it is nearly supersymmetric, it likely cannot support life, because of the implied near-symmetry between matter and forces. These two assumptions imply that the most likely place to be in the multiverse is in the habitable vacuum that can be reached via the fastest decay process from the master vacuum.

We know that in the past our universe had a high Hubble rate, and a high energy scale. To reach a universe like ours from the master vacuum, a large violation of the Null Energy Condition (NEC) must have occurred [5]. Thus, the fastest possible NEC violation will play a crucial role in the multiverse. Presently, several conjectures exist as to how the NEC can be violated in a cosmological context. These conjectures can be subdivided into two broad categories, namely quantum NEC violations and classical ones. The best-understood quantum NEC violation is that of up-tunneling, where an extended spacetime region in de Sitter space can perform a quantum jump (described by a Coleman-de Luccia instanton) to a higher-Hubble-rate de Sitter vacuum [6]. This process is reasonably well understood in semiclassical quantum gravity, but it is ultra-slow,
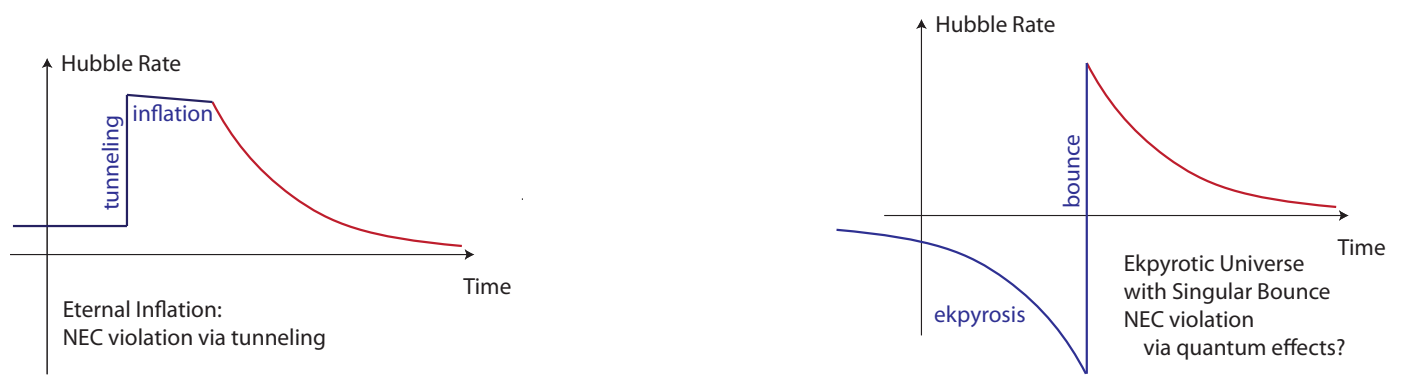

Figure 1: Quantum violations of the Null Energy Condition: on the left via uptunneling, and on the right via a quantum resolution of a classical big crunch singularity. 

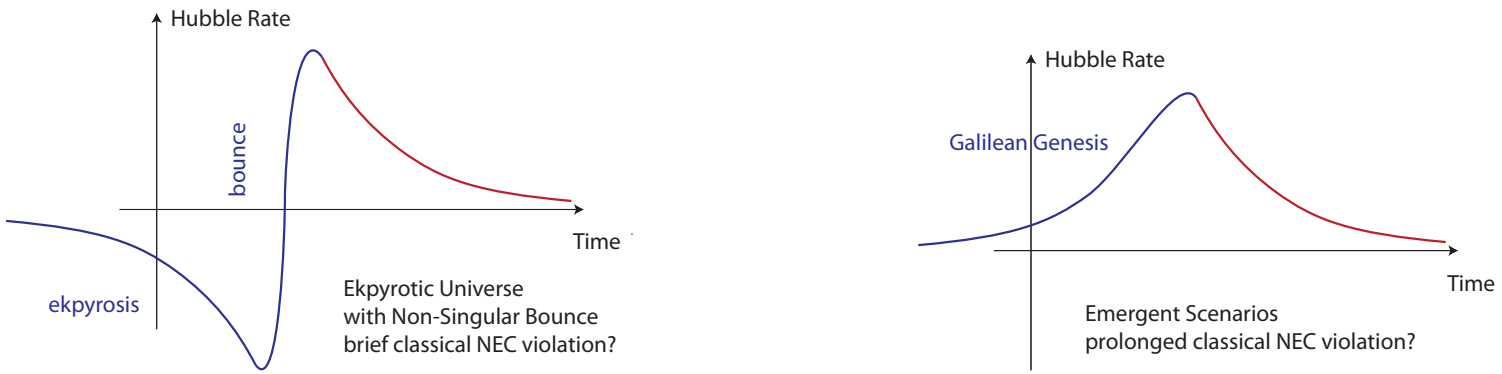

Figure 2: Classical violations of the NEC: on the left a non-singular bounce such as envisioned in new ekpyrotic cosmology, and on the right the emergent phase in the Galilean genesis scenario.

and will only be truly relevant if no faster NEC-violating processes exist. A second such quantum violation of the NEC is the conjectured quantum resolution of classically singular big crunch/big bang transitions [7]. If feasible, such processes will have dramatic consequences for cosmology, see e.g. [8].

One may wonder whether it is also possible to have classical violations of the NEC. Here, very few models are currently available: the only known scalar-field models are the ghost condensate [9] and the closely related Galileons [10] - both of these involve higher derivatives in an essential manner. These theories have already led to new cosmological models, such as new ekpyrotic cosmology [11] or Galilean genesis [12], which, if consistent, would play an important role in a multiverse context [5]. What is not clear at present is whether such models are truly consistent, and whether or not they can be embedded into a UV complete framework. As a first step in performing such an analysis, we will construct a supergravitational version of the ghost condensate model below.

The plan of this contribution is as follows: we will first introduce our formalism for constructing chiral superfield actions with higher derivatives, first in global and then in local supersymmetry. We will then look at the elimination of the auxiliary fields, especially the auxiliary field $F$ that is contained in chiral multiplets, and show that this leads to new expressions for the scalar field potentials. When the higher-derivative terms are small, we obtain correspondingly small corrections to both the kinetic and potential terms. For large higher-derivative terms, the corrections are crucial, and a generic effect that we find is that the potential becomes negative in this regime. Thus a simple implementation of DBI inflation actually fails. We subsequently show how to overcome this limitation by coupling the model to an additional chiral superfield. Finally, we look at the ghost condensate model in supergravity, and discuss its properties, such as its ability to break local supersymmetry without the super-Higgs effect taking place. We will conclude with a short discussion.

\section{Higher-derivative chiral superfield actions in $\mathscr{N}=1$ supergravity}

\subsection{Higher-derivative chiral superfields in global supersymmetry}

We begin by considering global $\mathscr{N}=1$ supersymmetry and will work in superspace. Our notations and conventions are those of Wess and Bagger [13]. A chiral superfield $\Phi$, defined by the 
constraint $\bar{D} \Phi=0$, has the expansion

$$
\Phi=A(x)+\sqrt{2} \theta \chi(x)+\theta \theta F(x)+\mathrm{i} \theta \sigma^{m} \bar{\theta} \partial_{m} A(x)-\frac{\mathrm{i}}{\sqrt{2}} \theta \theta \partial_{m} \chi(x) \sigma^{m} \bar{\theta}+\frac{1}{4} \theta \theta \bar{\theta} \bar{\theta} \square A(x),
$$

where $A$ is a complex scalar, $\chi_{\alpha}$ is a spin- $\frac{1}{2}$ fermion and $F$ is a complex auxiliary field. The component expansion (2.1) can be simplified by using the coordinates $y^{m}=x^{m}+\mathrm{i} \theta \sigma^{m} \bar{\theta}$, in terms of which

$$
\Phi=A(y)+\sqrt{2} \theta \chi(y)+\theta \theta F(y) .
$$

This form of the component expansion has a straightforward generalization to curved superspace.

A general feature of superspace is that the highest component (that is, the $\theta \theta \bar{\theta} \bar{\theta}$ component) transforms under supersymmetry into a total spacetime derivative. Thus, the highest component of a superfield, which can be isolated by integrating over superspace, can be used to construct a supersymmetric Lagrangian.

In [14], it was shown how to construct supersymmetric actions involving higher-derivatives of chiral superfields. The construction is based on a particular supersymmetric extension of the scalar field Lagrangian $(\partial \phi)^{4}$ given by $D^{\alpha} \Phi D_{\alpha} \Phi \bar{D}_{\dot{\alpha}} \Phi^{\dagger} \bar{D}^{\dot{\alpha}} \Phi^{\dagger}$. Ignoring the fermion $\chi$, this superfield contains only the $\theta \theta \bar{\theta} \bar{\theta}$ component

$$
D^{\alpha} \Phi D_{\alpha} \Phi \bar{D}_{\dot{\alpha}} \Phi^{\dagger} \bar{D}^{\dot{\alpha}} \Phi^{\dagger}=\theta \theta \bar{\theta} \bar{\theta}\left(16(\partial A)^{2}\left(\partial A^{*}\right)^{2}-32|\partial A|^{2}|F|^{2}+16|F|^{4}\right),
$$

where the complex scalar $A$ is composed of two real scalars $\phi, \xi$ as

$$
A=\frac{1}{\sqrt{2}}(\phi+\mathrm{i} \xi)
$$

and $|\partial A|^{2} \equiv \partial A \cdot \partial A^{*}$. Thus, the superspace integral of the superfield (2.3) yields the term

$$
16(\partial A)^{2}\left(\partial A^{*}\right)^{2}=4(\partial \phi)^{4}+4(\partial \xi)^{4}-8(\partial \phi)^{2}(\partial \xi)^{2}+16(\partial \phi \cdot \partial \xi)^{2}
$$

plus terms involving the auxiliary field $F$. Hence, Eq. (2.3) constitutes a possible supersymmetric extension of $(\partial \phi)^{4}$. This superfield possesses several particularly useful properties: 1) It constitutes a supersymmetric extension of the precise expression $(\partial \phi)^{4}$, and does not contain other terms involving $\phi$ alone. 2) Despite the higher-derivative nature of the superfield, the auxiliary field $F$ does not obtain a kinetic energy. This is non-trivial, as on dimensional grounds a term such as $|A|^{2}|\partial F|^{2}$ could have arisen, and implies that $F$ remains truly auxiliary. 3) The auxiliary field now appears at quartic order in the action and, thus, its equation of motion is cubic. Hence, in contrast to the usual two-derivative supersymmetric theories, there exist now up to three different solutions for $F$. 4) Finally, the most crucial property for our present purposes is the fact that the bosonic part of $D^{\alpha} \Phi D_{\alpha} \Phi \bar{D}_{\dot{\alpha}} \Phi^{\dagger} \bar{D}^{\dot{\alpha}} \Phi^{\dagger}$, given in (2.3), only contains a non-zero top $\theta \theta \bar{\theta} \bar{\theta}$ component- all lower components vanish. It follows that if one multiplies this superfield with any function $T$ of $\Phi, \Phi^{\dagger}$ and (an arbitrary number of) their spacetime derivatives, then the component expansion will be given by (2.3) times $T \mid$, where inside $T \mid$ the chiral superfield $\Phi$ is simply replaced by its lowest component $A$. This allows one to easily construct a supersymmetric extension of any higherderivative scalar Lagrangian containing $(\partial \phi)^{4}$ as a factor, simply by performing the replacement $\phi \rightarrow \sqrt{2} A \rightarrow \sqrt{2} \Phi$ in the co-factor. 
This last property allows one to construct a supersymmetric extension of theories with Lagrangian $P(X, \phi)$, where $X \equiv-\frac{1}{2}(\partial \phi)^{2}$. Specifically, for $P(X, \phi)=\sum_{n \geq 1} a_{n}(\phi) X^{n}$, the higherderivative terms in the supersymmetric generalization are the $\mathrm{d}^{2} \theta \mathrm{d}^{2} \bar{\theta}$ integral of

$$
\begin{gathered}
\frac{1}{16} D \Phi D \Phi \bar{D} \Phi^{\dagger} \bar{D} \Phi^{\dagger} T\left(\Phi, \Phi^{\dagger}, \partial_{m} \Phi, \partial_{n} \Phi^{\dagger}\right), \\
\text { where } \quad T\left(\Phi, \Phi^{\dagger}, \partial_{m} \Phi, \partial_{n} \Phi^{\dagger}\right)=\sum_{n \geq 2} a_{n}\left(\frac{1}{4} \partial^{m}\left(\Phi+\Phi^{\dagger}\right) \partial_{m}\left(\Phi+\Phi^{\dagger}\right)\right)^{n-2}
\end{gathered}
$$

with $a_{n}=a_{n}\left(\frac{\Phi+\Phi^{\dagger}}{\sqrt{2}}\right)$. Particular applications are a supersymmetric form of the DBI action [14], as well as a supersymmetric ghost condensate theory- both in flat spacetime. However, the most interesting phenomenological consequences occur when these models are coupled to gravity- for example, inflation driven by the DBI part of the action or cosmic bounces induced by a ghost condensate. It is, therefore, of interest to include gravity in the analysis. In a supersymmetric context, this means extending the above construction to curved superspace. This will be the topic of the next section.

\subsection{Extending to supergravity}

We now want to extend the above results to $\mathscr{N}=1$ supergravity. As with global supersymmetry, supergravity is most easily expressed in superspace-now, however, with non-vanishing curvature. In this case, one can introduce new fermionic coordinates $\Theta$ which are defined precisely so that the $(A, \chi, F)$ components of a chiral superfield $\Phi$ arise as the coefficients of the expansion

$$
\Phi=A+\sqrt{2} \Theta^{\alpha} \chi_{\alpha}+\Theta^{\alpha} \Theta_{\alpha} F .
$$

In curved superspace, supersymmetric Lagrangians can be constructed from the chiral integrals

$$
\int \mathrm{d}^{2} \Theta\left(\overline{\mathscr{D}}^{2}-8 R\right) L
$$

where $L$ is a scalar, hermitean function. Note that the chiral projector in curved superspace is $\overline{\mathscr{D}}^{2}-8 R$, where $\overline{\mathscr{D}}_{\dot{\alpha}}$ is a spinorial component of the curved superspace covariant derivative $\mathscr{D}_{A}=$ $\left(\mathscr{D}_{a}, \mathscr{D}_{\alpha}, \overline{\mathscr{D}}_{\dot{\alpha}}\right)$ and $R$ is the curvature superfield. In its component expansion, $R$ contains the Ricci scalar $\mathscr{R}$ and the gravitino $\psi_{m}$, as well as the auxiliary fields of supergravity- namely a complex scalar $M$ and a real vector $b_{m}$. A second superfield that we will need is the chiral density $\mathscr{E}$ - this contains the determinant of the vierbein $e$, as well as $M$ and $\psi_{m}$. For most of this contribution, we will set the fermions to zero.

We introduce a hermitean Kähler potential $K\left(\Phi^{i}, \Phi^{\dagger k *}\right)$ of the chiral superfields $\Phi^{i}$ (where $i$ enumerates the fields), along with a holomorphic superpotential $W\left(\Phi^{i}\right)$. Their Lagrangian is

$$
\mathscr{L}=\int \mathrm{d}^{2} \Theta 2 \mathscr{E}\left[\frac{3}{8}\left(\overline{\mathscr{D}}^{2}-8 R\right) e^{-K\left(\Phi^{i}, \Phi^{\dagger k *}\right) / 3}+W\left(\Phi^{i}\right)\right]+\text { h.c. }
$$

We now add the higher-derivative kinetic terms for the chiral superfields, now, however, in a manifestly diffeomorphism invariant manner $[15,16]$. Specifically, we introduce

$$
\begin{aligned}
\mathscr{L}_{\mathrm{h}-\mathrm{d}} & =-\frac{1}{8} \int \mathrm{d}^{2} \Theta 2 \mathscr{E}\left(\overline{\mathscr{D}}^{2}-8 R\right) \mathscr{D} \Phi^{i} \mathscr{D} \Phi^{j} \overline{\mathscr{D}} \Phi^{\dagger k *} \overline{\mathscr{D}} \Phi^{\dagger l *} T_{i j k * l *}+h . c . \\
& =16 e\left[\left(\partial A^{i} \cdot \partial A^{j}\right)\left(\partial A^{k *} \cdot \partial A^{l *}\right)-2 F^{i} F^{k *}\left(\partial A^{j} \cdot \partial A^{l *}\right)+F^{i} F^{j} F^{k *} F^{l *}\right] T_{i j k * l *} \mid,
\end{aligned}
$$


where $T_{i j k * l *} \mid$ is the lowest component of the tensor superfield $T_{i j k * l *}$. Let us clarify the meaning of $T_{i j k * l *}$. First, this superfield transforms as a four-index tensor on the Kähler manifold in which the scalar fields take their values and, thus, ensures target space diffeomorphism invariance. Second, $T_{i j k * l *}$ is required to be hermitian and symmetric in the pair of indices $i, j$ as well as in $k^{*}, l^{*}$. Third, any tensor satisfying these constraints can be multiplied by an arbitrary real function of the chiral superfields and an unlimited number of their $\mathscr{D}_{m}$ covariant derivatives, as long as all indices stemming from the covariant derivatives are contracted. The simplest example of $T_{i j k * l *}$ is $\frac{1}{2}\left(g_{i k *} g_{j l *}+g_{i l *} g_{j k *}\right)$, where $g_{i j^{*}}$ is the Kähler metric. We would like to stress that the fact that one can multiply this tensor with an arbitrary function of the chiral superfields and their spacetime derivatives means that we can obtain a supergravity extension of any term that involves $(\partial \phi)^{4}$ as a factor. An illustrative example of the usefulness of this property is provided by the DBI action presented in Section 3.

The sum of the two actions Eqs. (2.10)+(2.11) does not lead to ordinary Einstein frame gravity but, rather, to a scalar-gravity theory of the form $e^{-K / 3} \mathscr{R}$. One can transform the action into Einstein frame by performing the Weyl rescaling

$$
e_{n}^{a} \rightarrow e_{n}{ }^{a} e^{K / 6} .
$$

Note that the higher-derivative term does not contribute to the gravity-scalar coupling and, hence, we can perform the same Weyl rescaling as in ordinary chiral supergravity without higher-derivatives. This is a non-trivial feature of our framework, which greatly facilitates subsequent calculations. After Weyl rescaling and the elimination of the auxiliary files $b_{m}$ and $M$, the Lagrangian becomes

$$
\begin{aligned}
\frac{1}{e} \mathscr{L}_{\text {Weyl }}= & -\frac{1}{2} \mathscr{R}-g_{i k *} \partial A^{i} \cdot \partial A^{k *}+g_{i k *} e^{K / 3} F^{i} F^{k *}+e^{2 K / 3}\left[F^{i}\left(D_{A} W\right)_{i}+F^{k *}\left(D_{A} W\right)_{k *}^{*}\right]+3 e^{K} W W^{*} \\
& +16\left[\left(\partial A^{i} \cdot \partial A^{j}\right)\left(\partial A^{k *} \cdot \partial A^{l *}\right)-2 F^{i} F^{k *}\left(\partial A^{j} \cdot \partial A^{l *}\right)+F^{i} F^{j} F^{k *} F^{l *}\right] T_{i j k * l * \text { Weyl }} \mid \cdot \quad \text { (2.13) }
\end{aligned}
$$

In the next section, we will discuss the remaining auxiliary field, namely $F$, in detail.

\subsection{New expressions for potentials after eliminating the auxiliary field $F$}

We now consider the most interesting of the auxiliary fields, namely $F$. Its equation of motion is

$$
g_{i k *} F^{i}+e^{K / 3}\left(D_{A} W\right)_{k *}^{*}+32 F^{i}\left(e^{K / 3} F^{j} F^{l *}-\partial A^{j} \cdot \partial A^{l *}\right) T_{i j k * l * \text { Weyl }} \mid=0 .
$$

This equation is now cubic in $F$ and, thus, it can have up to three inequivalent solutions. As we will see, these different solutions lead to different theories! Here, we will restrict our analysis to a single chiral superfield $\Phi^{1}=\Phi$, the extension to multiple superfields being straightforward to implement. In this case, the equation of motion for $F$ becomes

$$
K_{, A A^{*}} F+e^{K / 3}\left(D_{A} W\right)^{*}+32 F\left(e^{K / 3}|F|^{2}-|\partial A|^{2}\right) \mathscr{T}=0,
$$

where we use the simplified notation

$$
\mathscr{T} \equiv T_{111 * 1 * \text { Weyl }} \mid
$$

Note that $\mathscr{T}$ is effectively an arbitrary real scalar function of $A, A^{*}$ and their spacetime covariant derivatives $\mathscr{D}_{m} \ldots \partial_{n} A, \mathscr{D}_{m} \ldots \partial_{n} A^{*}$. Multiplying (2.15) with $F^{*}$ shows that $\left(D_{A} W\right)^{*} F^{*}$ must be real. 
Thus, one can relate $F$ and $F^{*}$ via $F^{*}=F D_{A} W /\left(D_{A} W\right)^{*}$ as long as $\left(D_{A} W\right)^{*} \neq 0$, which we now assume. One can use this relation to obtain a cubic equation for $F$ alone. This is given by

$$
K_{, A A^{*}} F+e^{K / 3}\left(D_{A} W\right)^{*}+32\left(e^{K / 3} \frac{D_{A} W}{\left(D_{A} W\right)^{*}} F^{3}-|\partial A|^{2} F\right) \mathscr{T}=0 .
$$

In general, this equation admits three distinct solutions- which we denote by $F_{1}, F_{2}, F_{3}$-leading to three different theories. One can find these solutions using Cardano's formula. Define

$$
p=e^{-K / 3} \frac{\left(D_{A} W\right)^{*}}{D_{A} W}\left(\frac{K_{, A A^{*}}}{32 \mathscr{T}}-|\partial A|^{2}\right), \quad q=\frac{1}{32 \mathscr{T}} \frac{\left(D_{A} W\right)^{* 2}}{D_{A} W}, \quad D=\left(\frac{q}{2}\right)^{2}+\left(\frac{p}{3}\right)^{3} .
$$

Then the solutions are given by

$$
F_{k+1}=\omega^{k} F_{+}+\omega^{-k} F_{-},
$$

where $k=0,1,2, \omega=e^{2 \pi \mathrm{i} / 3}=-\frac{1}{2}+\mathrm{i} \frac{\sqrt{3}}{2}$ is a cube root of unity and $F_{ \pm}=\left(-\frac{q}{2} \pm D^{1 / 2}\right)^{1 / 3}$. Substituting these solutions back into the action generates three different branches of the theory. Only one of these solutions approaches the usual solution for $F$ when $\mathscr{T} \rightarrow 0$ - we call this solution the ordinary branch, and we will focus on this solution here. For more details, see [15].

The higher-derivative terms are all proportional to the $\mathscr{T}$ tensor. Therefore, by assuming that $\mathscr{T}$ contains a factor that can be tuned to be small, one can treat such terms as sub-leading. The $\mathscr{T} \rightarrow 0$ limit then corresponds to $q \ll p^{3 / 2}$, which for the ordinary branch implies

$$
\begin{aligned}
F_{1}= & -K^{, A A^{*}} e^{K / 3}\left(D_{A} W\right)^{*} \\
& +32 \mathscr{T} e^{4 K / 3}\left(K^{, A A^{*}}\right)^{4}\left(D_{A} W\right)^{* 2} D_{A} W-32 \mathscr{T} e^{K / 3}\left(K^{, A A^{*}}\right)^{2}\left(D_{A} W\right)^{*}|\partial A|^{2}+\mathscr{O}\left(\mathscr{T}^{2}\right)
\end{aligned}
$$

Note that this corresponds to a small correction to the usual solution for the auxiliary field $F$ in the presence of a superpotential. Correspondingly, we obtain small corrections in the Lagrangian by substituting this solution for $F$. To first order in the higher-derivative terms, we get

$$
\begin{gathered}
\frac{1}{e} \mathscr{L}_{\text {ordinary }, \mathscr{T} \rightarrow 0}=-\frac{1}{2} \mathscr{R}-K_{, A A^{*}}|\partial A|^{2}-32 e^{K} K^{, A A^{*}}\left|D_{A} W\right|^{2} K^{, A A^{*}}|\partial A|^{2} \mathscr{T} \\
+16(\partial A)^{2}\left(\partial A^{*}\right)^{2} \mathscr{T}-e^{K}\left(K^{, A A^{*}}\left|D_{A} W\right|^{2}-3|W|^{2}\right)+16 e^{2 K}\left(K^{, A A^{*}}\left|D_{A} W\right|^{2}\right)^{2}\left(K^{, A A^{*}}\right)^{2} \mathscr{T} .
\end{gathered}
$$

An interesting feature is that both the kinetic terms and the potential get corrected. The potential now becomes

$$
V=e^{K}\left(K^{, A A^{*}}\left|D_{A} W\right|^{2}-3|W|^{2}\right)-16\left(e^{K} K^{, A A^{*}}\left|D_{A} W\right|^{2}\right)^{2}\left(K^{, A A^{*}}\right)^{2} \mathscr{T}_{\text {noder. }},
$$

where $\mathscr{T}_{\text {noder. }}$ stands for the part of $\mathscr{T}$ that does not contain spacetime derivatives. Note that all the correction terms in the Lagrangian above are invariant under Kähler transformations.

As an example, consider the case where $K=\Phi \Phi^{\dagger}, \mathscr{T}=\tau\left(K_{, A A^{*}}\right)^{2}$ is of canonical form with $\tau$ a small parameter and $W=\Phi$. Then the potential, to first order in $\tau$, is given by $V=\bar{V}+\delta V$. Near the minimum at $A=0$ the potential can be approximated by

$$
\bar{V} \approx 1+\frac{1}{2}|A|^{4}+\cdots
$$


Note that the $|A|^{2}=\phi^{2}+\xi^{2}$ term cancels in the expansion. Therefore, this potential is very flat near the origin, rising only quartically as $\left(\phi^{2}+\xi^{2}\right)^{2}$. The leading order correction to this potential is given by

$$
\delta V \approx-16 \tau\left(1+6|A|^{2}+16|A|^{4}+\cdots\right) .
$$

For $\frac{1}{128}>\tau>0$, the minimum at $A=0$ becomes a local maximum. The potential is now minimized along a circle defined by $|A|^{2}=12 \tau /(1-128 \tau)$. In other words, the potential changes from a slowly rising quartic potential with a minimum at the origin to a "Mexican hat".

\section{DBI Inflation in supergravity - an example of higher-derivative dominance}

Using our formalism, we can write a supergravity version of the single real scalar field DBI action [17]. It turns out that we need to consider a canonical Kähler potential $K_{, A A^{*}}=1$ and a tensor superfield [18]

$$
16 T=\frac{f\left(\Phi, \Phi^{\dagger}\right)}{1+f \partial \Phi \cdot \partial \Phi^{\dagger} e^{K / 3}+\sqrt{\left(1+f \partial \Phi \cdot \partial \Phi^{\dagger} e^{K / 3}\right)^{2}-f^{2}(\partial \Phi)^{2}\left(\partial \Phi^{\dagger}\right)^{2} e^{2 K / 3}}} .
$$

Here $f\left(\Phi, \Phi^{\dagger}\right)$ is an arbitrary hermitian function and we have used the notation that $\partial \Phi \cdot \partial \Phi^{\dagger}=$ $g^{m n} \mathscr{D}_{m} \Phi \mathscr{D}_{n} \Phi^{\dagger}$. In a brane setting, the lowest component of the $f$ function can be identified with the warp factor of the direction in which the brane moves. This leads to the Lagrangian

$$
\begin{aligned}
\frac{1}{e} \mathscr{L}= & -\frac{1}{2} \mathscr{R}+3 e^{K}|W|^{2}-\frac{1}{f}\left(\sqrt{1+2 f \partial A \cdot \partial A^{*}+f^{2}\left(\partial A \cdot \partial A^{*}\right)^{2}-f^{2}(\partial A)^{2}\left(\partial A^{*}\right)^{2}}-1\right) \\
& +e^{K / 3}|F|^{2}+e^{2 K / 3}\left(F\left(D_{A} W\right)+F^{*}\left(D_{A} W\right)^{*}\right)-32 e^{K / 3}|F|^{2} \partial A \cdot \partial A^{*} \mathscr{T}+16 e^{2 K / 3}|F|^{4} \mathscr{T} .
\end{aligned}
$$

Here $\mathscr{T}$, which is the Weyl rescaled lowest component of $T$, is given by

$$
16 \mathscr{T}=\frac{f}{1+f \partial A \cdot \partial A^{*}+\sqrt{\left(1+f \partial A \cdot \partial A^{*}\right)^{2}-f^{2}(\partial A)^{2}\left(\partial A^{*}\right)^{2}}}
$$

with $f=f\left(A, A^{*}\right)$. The last term in the first line of (3.2) can be recognized as the DBI action for the two real scalar fields $\phi, \xi$ that make up the complex scalar $A$. That is, the simplest $\mathscr{N}=1$ supergravity generalization of the single real scalar DBI action naturally produces a DBI theory for both real scalar component fields. As can be seen from the action, when the fields depend only on time there exists an upper bound on the velocity of $A$ given by

$$
|\dot{A}|^{2} \leq \frac{1}{2 f}
$$

The so-called relativistic regime corresponds to the situation where this bound is (almost) saturated. Models of DBI inflation [1] exploit this inequality. As the brane moves towards a region of large $f$, the scalars are automatically constrained to move slowly-allowing for inflation to occur on potentials that would otherwise be too steep.

In the above Lagrangian, the auxiliary field $F$ has not yet been eliminated. When $f$ is small, so is $\mathscr{T}$ and in the ordinary branch $F$ approaches the usual solution

$$
F \approx-e^{K / 3}\left(D_{A} W\right)^{*} . \quad(f \text { small })
$$


In this non-relativistic limit, after substituting for $F$ one obtains the usual potential

$$
V_{\text {non-rel. }}=e^{K}\left(\left|D_{A} W\right|^{2}-3|W|^{2}\right) .
$$

Note that this expression is only valid as long as the higher-derivative terms in $A$ are irrelevant. More interesting for our purposes is the relativistic limit, where $f$ is large and $|\dot{A}|^{2}$ correspondingly small, with $\mathscr{T} \approx f / 8$. In that case, the solution for $F$ approaches [19]

$$
F \approx-\left(\frac{\left(D_{A} W\right)^{* 2}}{4 f D_{A} W}\right)^{1 / 3} \cdot \quad(f \text { large })
$$

After substituting for $F$ in the relativistic limit, the Lagrangian becomes [17]

$$
\begin{aligned}
\frac{1}{e} \mathscr{L}_{\text {rel. }}= & -\frac{1}{2} \mathscr{R}+3 e^{K}|W|^{2}-\frac{3}{2} \frac{e^{K}\left|D_{A} W\right|^{2}}{\left(4 f e^{K}\left|D_{A} W\right|^{2}\right)^{1 / 3}} \\
& -\frac{1}{f}\left(\sqrt{1+2 f \partial A \cdot \partial A^{*}+f^{2}\left(\partial A \cdot \partial A^{*}\right)^{2}-f^{2}(\partial A)^{2}\left(\partial A^{*}\right)^{2}}-1\right)+\mathscr{O}\left(f^{-2 / 3}\right) .
\end{aligned}
$$

Thus, to leading order the potential is given by

$$
V_{\text {rel. }}=-3 e^{K}|W|^{2}
$$

which is negative for any choice of superpotential. The term arising from eliminating $F$ is subleading. It is evident, therefore, that inflation cannot occur since a phase of de-Sitter-like expansion requires a positive energy density in the universe. Thus, supergravitational relativistic DBI inflation with a single chiral superfield does not work!

Let us now extend this theory by coupling it to a second chiral superfield $S$ with component expansion

$$
S=B+\Theta^{\alpha} \Theta_{\alpha} F_{B}
$$

Here $B$ is a complex scalar and $F_{B}$ the complex auxiliary field associated with $S$. We will take this second field to have a two-derivative action. Then, choosing a Kähler potential such that $K_{, A A^{*}}=1, K_{, A B^{*}}=0=K_{, A^{*} B}$, and after the same manipulations as in the previous section- Weyl rescaling the action and eliminating the auxiliary fields $b_{m}, M$-we obtain the Lagrangian

$$
\begin{aligned}
\frac{1}{e} \mathscr{L}= & -\frac{1}{2} \mathscr{R}+3 e^{K}|W|^{2}-K_{, B B^{*}} \partial B \cdot \partial B^{*} \\
& -\frac{1}{f}\left(\sqrt{1+2 f \partial A \cdot \partial A^{*}+f^{2}\left(\partial A \cdot \partial A^{*}\right)^{2}-f^{2}(\partial A)^{2}\left(\partial A^{*}\right)^{2}}-1\right) \\
& +K_{, B B^{*}} e^{K / 3}\left|F_{B}\right|^{2}+e^{2 K / 3}\left(F_{B}\left(D_{B} W\right)+F_{B}^{*}\left(D_{B} W\right)^{*}\right) \\
& +e^{K / 3}|F|^{2}+e^{2 K / 3}\left(F\left(D_{A} W\right)+F^{*}\left(D_{A} W\right)^{*}\right)-32 e^{K / 3}|F|^{2} \partial A \cdot \partial A^{*} \mathscr{T}+16 e^{2 K / 3}|F|^{4} \mathscr{T} .
\end{aligned}
$$

In this expression, the auxiliary fields $F, F_{B}$ of the two chiral multiplets have not yet been eliminated. Their equations of motion are given by

$$
\begin{aligned}
& F+e^{K / 3}\left(D_{A} W\right)^{*}+32 F \mathscr{T}\left(e^{K / 3}|F|^{2}-\partial A \cdot \partial A^{*}\right)=0, \\
& K_{, B B^{*}} F_{B}+e^{K / 3}\left(D_{B} W\right)^{*}=0
\end{aligned}
$$


Note that these equations are not coupled and, thus, $F$ can be eliminated as in the previous section. It is also straightforward to substitute for $F_{B}$, since its equation of motion is algebraic and linear. In the non-relativistic limit-that is, when $f$ is small-one obtains the usual potential

$$
V_{\text {non-rel.,2 superfields }}=e^{K}\left(\left|D_{A} W\right|^{2}+K^{, B B^{*}}\left|D_{B} W\right|^{2}-3|W|^{2}\right) .
$$

However, in the relativistic limit the $\left|D_{A} W\right|^{2}$ term again is subdominant and the potential becomes

$$
V_{\text {rel.,2 } 2 \text { superfields }}=e^{K}\left(K^{, B B^{*}}\left|D_{B} W\right|^{2}-3 e^{K}|W|^{2}\right) .
$$

Comparing this to expression Eq. (3.9), we see that in the two superfield case a new, positive definite term enters the potential energy! Hence, by choosing the superpotential appropriately, the overall potential can be made positive along the direction(s) of interest in field space-thus enabling inflation to occur.

We choose for the superpotential $W$ an Ansatz first used in [20] and analyzed, in detail, in [21] within the context of ordinary two-derivative supergravity. This Ansatz is

$$
W=S w(\Phi),
$$

where $w(\Phi)$ is a "real" holomorphic function of $\Phi$; that is, $w(\Phi)=\sum_{n} c_{n} \Phi^{n}$ with $c_{n} \in \mathbb{R}$. The coefficients are chosen to be real for simplicity. The lowest component of $W$ is given by $B w(A)$. On the $B=0$ plane, we have $W=0, D_{B} W=w(A)$ and, hence, the potential energy (3.15) becomes

$$
V_{B=0}=e^{K\left(A, A^{*}\right)} K^{, B B^{*}}|w(A)|^{2} .
$$

Here, the Kähler potential is also evaluated at $B=0$. The $B$ field can always be rescaled so that its kinetic term is canonical (when $B=0$ ). Correspondingly, we will take $\left.K_{, B B^{*}}\right|_{B=0}=1$. Then the potential further simplifies to the manifestly positive definite expression

$$
V_{B=0}=e^{K\left(A, A^{*}\right)}|w(A)|^{2} .
$$

For this expression to be physically relevant, one must ensure that the dynamics is restricted to the $B=0$ plane. As shown in [17], this leads to the stability condition $K_{, B B B^{*} B^{*}} \lesssim-\frac{1}{3}$. This condition is analogous to that found in two-derivative supergravity models [21]. One can also restrict the theory further, so that only a single real scalar field remains dynamical. For this purpose, choose the Kähler potential to depend on $\Phi, \Phi^{\dagger}$ via the combination $-\frac{1}{2}\left(\Phi-\Phi^{\dagger}\right)^{2}$ only. Then, the Kähler potential will not depend on $\phi$. Correspondingly, if $\xi$ is now stabilized around $\xi=0$, then the dynamics will take place entirely in the $\phi$ direction with the potential

$$
V_{\phi}=w\left(\frac{\phi}{\sqrt{2}}\right)^{2} .
$$

Thus, any smooth positive potential can be engineered in this way, simply by identifying $w$ with the square root of the desired potential and analytically continuing $w$ to the complex plane [21]. However, for consistency, one must check that $\xi$ is stabilized by a sufficiently high mass. As shown in [17], this translates into the requirement $K_{, A A^{*} B B^{*}} \lesssim \frac{5}{6}$. Examples of Kähler potentials satisfying all of the above assumptions and stability constraints were discussed in [21]. 
Now note that for the superpotential (3.16), $D_{A} W$ is proportional to $B$ and hence vanishes on the $B=0$ plane. Thus, in going from the approximately two-derivative regime to the relativistic DBI regime, the potential does not change for the models considered here. This special feature is entirely non-trivial, and arises as a direct consequence of the choice (3.16). It greatly facilitates the analysis of the corresponding inflationary models.

\section{A supergravitational ghost condensate}

Ghost condensate vacua [9] arise in higher-derivative theories of the form $\mathscr{L}=\sqrt{-g} P(X)$, where $P(X)$ is a differentiable function of $X$ containing an extremum. In a flat Friedmann-RobertsonWalker (FRW) spacetime with metric $d s^{2}=-d t^{2}+a(t)^{2} \delta_{i j} d x^{i} d x^{j}$, and assuming $\phi$ to be dependent on time alone, the scalar equation of motion becomes

$$
\frac{d}{d t}\left(a^{3} P_{, X} \dot{\phi}\right)=0 .
$$

Clearly this has a trivial solution when $\phi=$ constant. Of more interest is the solution with nonconstant $\phi$, but for which $X=\frac{1}{2} \dot{\phi}^{2}=$ constant, and $P_{, X}=0$. Denoting by $X_{\text {ext }}$ a constant extremum of $P(X)$, the equation of motion admits the ghost condensate solution

$$
\phi=c t
$$

where $c^{2}=2 X_{\text {ext }}$. The explicit time-dependence of this solution spontaneously breaks Lorentz invariance and leads to a number of interesting properties. First of all, evaluating the energy and pressure densities one finds

$$
\rho=2 X P_{, X}-P, \quad p=P \quad \Rightarrow \quad \rho+p=2 X P_{, X} .
$$

Since by definition $X>0$, it follows that the NEC can be violated if $P_{, X}<0$. That is, if we are close to an extremum for $P(X)$, then on one side the NEC is satisfied while on the other it is not. Correspondingly, since Einstein's equations imply $\dot{H}=-\frac{1}{2}(\rho+p)$, it is now possible to obtain a non-singular bouncing universe-where $H$ increases from negative to positive values. Crucial in determining the viability of this theory is the question of whether or not this NEC-violating solution is "stable". To this end, let us expand the Lagrangian to quadratic order in perturbations around the ghost condensate, $\phi=c t+\delta \phi\left(x^{m}\right)$. We find that

$$
\frac{\mathscr{L}}{\sqrt{-g}}=\frac{1}{2}\left(\left(2 X P_{, X X}+P_{, X}\right)(\dot{\delta} \phi)^{2}-P_{, X} \delta \phi^{, i} \delta \phi_{, i}\right)
$$

As a result of Lorentz breaking, the coefficients in front of the time and space pieces are unequal. By inspection, one sees that the condition for the absence of ghosts is that $2 X P_{, X X}+P_{, X}>0$, which can be achieved around a local minimum $P_{, X X}>0$ even in the NEC-violating region where $P_{, X}$ is small but negative. This feature is arguably the most striking property of ghost condensate theories, namely, that the NEC can be violated without the appearance of ghosts.

However, in the NEC violating region the coefficient in front of the spatial derivative term in (4.4) has the wrong sign. Therefore, the theory suffers from gradient instabilities. These can be 
softened by adding (small) higher-derivative terms-not of the $P(X)$ type-to the Lagrangian, such as $-(\square \phi)^{2}$. These modify the dispersion relation for $\delta \phi$ at high momenta and suppress instabilities for a short-but sufficient-period of time. In a cosmological context, there are additional constraints arising from a study of the growth of cosmological perturbations, which imply that a non-singular bounce must be fast in order to avoid perturbations from becoming uncontrollably large [22, 23]. The bottom line is that bouncing universe solutions via a ghost condensate are admissible, but the bounce is required to occur on a fast time-scale-for more details, see [11].

The supergravity extension of the prototype scalar ghost condensate $P(X)=-X+X^{2}$ is the sum of (2.10) and (2.11), where we take $K\left(\Phi, \Phi^{\dagger}\right)=-\Phi \Phi^{\dagger}, \mathscr{T}=\frac{1}{16}$, [24]. That is,

$$
\mathscr{L}_{T=1 / 16, \text { Weyl }}^{S U G R A}=\frac{1}{8}\left[\int \mathrm{d}^{2} \Theta 2 \mathscr{E}\left(\overline{\mathscr{D}}^{2}-8 R\right)\left(3 \mathrm{e}^{\Phi \Phi^{\dagger} / 3}-\frac{1}{2^{4}}\left(\mathscr{D} \Phi \mathscr{D} \Phi \overline{\mathscr{D}} \Phi^{\dagger} \overline{\mathscr{D}} \Phi^{\dagger}\right)\right)\right]_{\mathrm{Weyl}}+\text { h.c. }
$$

It follows that the purely bosonic part of this Lagrangian is

$$
\begin{aligned}
\frac{1}{e} \mathscr{L}_{T=1 / 16, \text { Weyl }}^{S U G R A}= & -\frac{1}{2} \mathscr{R}+|\partial A|^{2}+(\partial A)^{2}\left(\partial A^{*}\right)^{2}+\ldots \\
= & -\frac{1}{2} \mathscr{R}+\frac{1}{2}(\partial \phi)^{2}+\frac{1}{4}(\partial \phi)^{4} \\
& +\frac{1}{2}(\partial \xi)^{2}+\frac{1}{4}(\partial \xi)^{4}-\frac{1}{2}(\partial \phi)^{2}(\partial \xi)^{2}+(\partial \phi \cdot \partial \xi)^{2}+\ldots
\end{aligned}
$$

The remaining terms in the Lagrangian are at least quadratic in the fermions $\chi, \psi_{m}$. The Einstein and gravitino equations can be solved in a flat FRW spacetime with a vanishing gravitino $\psi_{m}=0$. The $\phi, \xi$ and $\chi$ equations of motion continue to admit a ghost condensate vacuum of the form

$$
\phi=c t, \quad \xi=0, \quad \chi=0
$$

where, to be consistent with the coupling to dynamical $a(t)$, one must set $c=1$. The scale factor is that of a de Sitter spacetime, which-in its flat slicing-is given by

$$
a(t)=e^{ \pm \frac{1}{\sqrt{12}} t} .
$$

The choice of the \pm sign corresponds to an expanding or contracting space respectively; in this contribution, we focus on the expanding branch. As shown in [24], the expanding branch is stable subject to small fluctuations, if appropriate supersymmetric stabilizing terms are added. These terms, analogously to the $-(\square \phi)^{2}$ term discussed above, modify the dispersion relations of the scalars $\phi$ and $\xi$ such that all fluctuations are well-behaved and gradient instabilities are under control over short periods of time. Due to lack of space, we refer to [24] for a detailed exposition.

A particularly interesting aspect of the supergravitational ghost condensate is provided by the fermionic sector of the theory. The relevant part of Lagrangian (to quadratic order in fermions) was derived in [24] and shown to be

$$
\begin{aligned}
\frac{1}{e} \mathscr{L}_{T=1 / 16, \mathrm{Weyl}}^{S U G R A}= & \ldots+\frac{1}{2} \varepsilon^{k l m n}\left(\bar{\psi}_{k} \bar{\sigma}_{l} \tilde{\mathscr{D}}_{m} \psi_{n}-\psi_{k} \sigma_{l} \tilde{\mathscr{D}}_{m} \bar{\psi}_{n}\right) \\
& +\frac{\mathrm{i}}{2}\left(\chi \sigma^{m} \mathscr{D}_{m} \bar{\chi}+\bar{\chi} \bar{\sigma}^{m} \mathscr{D}_{m} \chi\right)\left(1+\frac{1}{2}(\partial \phi)^{2}\right)+\frac{\mathrm{i}}{2} \phi^{, m} \phi_{, n}\left(\bar{\chi} \bar{\sigma}^{n}\left(\mathscr{D}_{m} \chi\right)+\chi \sigma^{n}\left(\mathscr{D}_{m} \bar{\chi}\right)\right) \\
& +\frac{1}{2}\left(\chi \sigma^{m} \bar{\sigma}^{n} \psi^{p}+\bar{\chi} \bar{\sigma}^{m} \sigma^{n} \bar{\psi}^{p}\right)\left(g_{m p} \phi_{, n}+\frac{1}{2} g_{m n} \phi_{, p}(\partial \phi)^{2}-\frac{1}{2} g_{n p} \phi_{, m}(\partial \phi)^{2}\right)+\ldots
\end{aligned}
$$


where $g_{m n}$ is the FRW metric. For the time-dependent vev $\phi=t,(\partial \phi)^{2}=-1$. Hence, the first and second/third lines correspond to unmixed $\psi_{m}$ and $\chi$ kinetic energies respectively. However, the ghost condensate induces a mass mixing term between $\chi$ and $\psi_{m}$. Using $\sigma^{m} \bar{\sigma}^{n}=-g^{m n}+2 \sigma^{m n}$, the mass term can be rewritten as

$$
-\frac{1}{4} \phi_{, m}\left(\chi \sigma^{m} \bar{\sigma}^{n} \psi_{n}+\bar{\chi} \bar{\sigma}^{m} \sigma^{n} \bar{\psi}_{n}\right) .
$$

This mass mixing term can be removed by redefining the gravitino. As we will discuss below, the supersymmetry transformations suggest the field redefinition

$$
\psi_{m \alpha}=\tilde{\psi}_{m \alpha}-\frac{2 \mathrm{i}}{(\partial \phi)^{2}} \mathscr{D}_{m}\left(\phi_{, n} \sigma_{\alpha \dot{\alpha}}^{n} \bar{\chi}^{\dot{\alpha}}\right)
$$

in terms of which the quadratic fermion Lagrangian becomes

$$
\begin{aligned}
\frac{1}{e} \mathscr{L}_{T=1 / 16, \text { Weyl }}^{\text {SUGRA }}= & \ldots+\frac{1}{2} \varepsilon^{k l m n}\left(\tilde{\bar{\psi}}_{k} \bar{\sigma}_{l} \tilde{\mathscr{D}}_{m} \tilde{\psi}_{n}-\tilde{\psi}_{k} \sigma_{l} \tilde{\mathscr{D}}_{m} \tilde{\bar{\psi}}_{n}\right) \\
& +\frac{\mathrm{i}}{2}\left(\chi \sigma^{m} \mathscr{D}_{m} \bar{\chi}+\bar{\chi} \bar{\sigma}^{m} \mathscr{D}_{m} \chi\right)+\mathrm{i} \phi^{, m} \phi_{, n}\left(\bar{\chi} \bar{\sigma}^{n}\left(\mathscr{D}_{m} \chi\right)+\chi \sigma^{n}\left(\mathscr{D}_{m} \bar{\chi}\right)\right)+\ldots
\end{aligned}
$$

This Lagrangian describes a) a massless gravitino $\tilde{\psi}_{m}$ with Lorentz covariant kinetic energy and b) a massless fermion $\chi$ with kinetic terms whose Lorentz invariance is broken in the ghost condensate background. We note that after the field redefinition of the gravitino, the kinetic terms for $\chi$ now appear with an additional overall multiplicative factor of 2 .

Given this result, one can analyze the super-Higgs effect within the context of the supergravity ghost condensate. The variations of the fermions $\chi$ and $\psi_{m}$-in a bosonic background-are given by

$$
\begin{aligned}
\delta \chi & =\mathrm{i} \sqrt{2} \sigma^{m} \bar{\zeta} \partial_{m} A+\sqrt{2} e^{K / 6} \zeta F, \\
\delta \psi_{m} & =2\left(\mathscr{D}_{m}+\frac{1}{4}\left(K_{, A} \partial_{m} A-K_{, A^{*}} \partial_{m} A^{*}\right)\right) \zeta+\mathrm{i} e^{K / 2} W \sigma_{m} \bar{\zeta} .
\end{aligned}
$$

In standard two-derivative chiral theories coupled to supergravity spontaneous breaking of supersymmetry is achieved by choosing a non-vanishing $W$ for which 1 ) the potential energy is minimized at constant $A$, and 2) when evaluated at this minimum $F=-K^{A A^{*}} e^{K / 3}\left(D_{A} W\right)^{*} \neq 0$. The non-vanishing $F$-term in (4.14) then renders the $\chi$ transformation inhomogeneous, spontaneously breaking supersymmetry, while the transformation of a redefined gravitino $\tilde{\psi}_{m}$ vanishes. Therefore, $\chi$ is the massless Goldstone fermion while $\tilde{\psi}_{m}$ is the physical gravitino. Generically, $W \neq 0$ in the vacuum giving the gravitino a non-vanishing mass

$$
m_{3 / 2}=e^{K / 2}|W|
$$

In the process the Goldstone fermion $\chi$ gets "eaten" by the now massive gravitino. This is the super-Higgs effect.

Let us now return to the supergravity ghost condensate vacuum. In this case we have $W=0$, from which it follows that $F=0$. However, $A$ now develops a non-zero, linearly time-dependent vev $\langle A\rangle=\langle\phi\rangle / \sqrt{2}=c t / \sqrt{2}$, where we restore the dimension-two constant $c$. The $\chi$ transformation in (4.14) then becomes

$$
\delta \chi=\mathrm{i} \sqrt{2} \sigma^{m} \bar{\zeta} \partial_{m} A=\mathrm{i} \sigma^{0} \bar{\zeta} c
$$


As previously, the fermion transforms inhomogeneously and, hence, supersymmetry is spontaneously broken. For the ghost condensate, however, the inhomogeneous term arises from the linear time-dependent vev of $\phi$ rather than from the $F$-term. Now consider the gravitino transformation (4.15), which here reduces to

$$
\delta \psi_{m}=2\left(\mathscr{D}_{m}+\frac{1}{4}\left(K_{, A} \partial_{m} A-K_{, A^{*}} \partial_{m} A^{*}\right)\right) \zeta=2 \mathscr{D}_{m} \zeta .
$$

Note that even though the factor $K_{, A} \partial_{m} A-K_{, A^{*}} \partial_{m} A^{*}$ vanishes in this vacuum, the de Sitter spacetime covariant derivative $\mathscr{D}_{m} \zeta_{\alpha}=\partial_{m} \zeta_{\alpha}-\frac{1}{2} \omega_{m p l}\left(\sigma^{p l}\right)_{\alpha} \beta \zeta_{\beta}$ does not vanish, as $\omega_{i 0 j}=g_{i j} H$, and, hence, $\psi_{m}$ transforms inhomogeneously. However, in analogy with the ordinary two-derivative case, let us redefine the gravitino as in (4.12). It is straightforward to shown that in the ghost condensate background

$$
\delta \tilde{\psi}_{m}=0 .
$$

This then identifies $\chi$ as the massless Goldstone fermion and $\tilde{\psi}_{m}$ as the physical gravitino. The generic expression for the gravitino mass was given by (4.16). In the ghost condensate, however, $W=0$ and, hence, $m_{3 / 2}=0$. That is, the breaking of local supersymmetry via a ghost condensate can occur without the gravitino obtaining a mass. This result for the supergravity ghost condensate is completely consistent with-and gives a physical explanation for-the preceding direct calculation of the quadratic fermion Lagrangian (4.14).

\section{Discussion and outlook}

The construction of supergravity coupled chiral superfield actions with higher derivatives has led to a number of surprises: 1) We have shown that these higher-derivative actions can be constructed in such a way that the auxiliary fields remain truly auxiliary. However, the auxiliary field $F$ that is associated with the chiral supermultiplets, now appear at quartic order in the action. Eliminating this field via its equation of motion in general now leads to three distinct branches of the theory, one of which is related to the usual solution for $F$. 2) Implementing DBI inflation in supergravity is harder than one might have thought: the higher-derivative terms, via their auxiliary fields, have a significant effect on the potential, and, generically, the potential becomes negative definite when the higher-derivative terms are important. We showed however how to circumvent this problem from a model-building point of view by coupling the theory to an additional chiral superfield with an ordinary kinetic term and an appropriate superpotential. 3) Perhaps surprisingly, we encountered no serious obstacle in constructing a supergravitational ghost condensate theory. This theory should allow one to explore in much more detail the relationship between supersymmetry and NEC violation.

There are many open questions for further research. Let us just mention a few: can such higherderivative supergravity models be derived by a direct string compactification? Can a full newekpyrotic model now be formulated in supergravity, combining the ordinary and ghost-condensate regimes? Can the present formalism be extended to a construction of other classes of supergravitational higher-derivative theories, and in particular to the Galileon theories (which were already extended to global supersymmetry in [25])? We hope to return to at least some of these questions in the future. 


\section{Acknowledgements}

I would like to thank my collaborators Matt Johnson, Justin Khoury, Michael Koehn and Burt Ovrut for many enjoyable and insightful discussions. I gratefully acknowledge the support of the European Research Council via the Starting Grant numbered 256994.

\section{References}

[1] E. Silverstein and D. Tong, Phys. Rev. D 70 (2004) 103505 [hep-th/0310221].

[2] C. Armendariz-Picon, T. Damour and V. F. Mukhanov, Phys. Lett. B 458 (1999) 209 [hep-th/9904075].

[3] B. Freivogel, Class. Quant. Grav. 28 (2011) 204007 [arXiv:1105.0244 [hep-th]].

[4] M. R. Douglas, arXiv:1204.6626 [hep-th].

[5] J. -L. Lehners, Phys. Rev. D 86 (2012) 043518 [arXiv:1206.1081 [hep-th]].

[6] K. -M. Lee and E. J. Weinberg, Phys. Rev. D 36 (1987) 1088.

[7] N. Turok, M. Perry and P. J. Steinhardt, Phys. Rev. D 70 (2004) 106004 [Erratum-ibid. D 71 (2005) 029901] [hep-th/0408083].

[8] P. J. Steinhardt and N. Turok, Phys. Rev. D 65 (2002) 126003 [hep-th/0111098]; J. -L. Lehners and P. J. Steinhardt, Phys. Rev. D 79 (2009) 063503 [arXiv:0812.3388 [hep-th]]; M. C. Johnson and J. -L. Lehners, Phys. Rev. D 85 (2012) 103509 [arXiv:1112.3360 [hep-th]].

[9] N. Arkani-Hamed, H. -C. Cheng, M. A. Luty and S. Mukohyama, JHEP 0405 (2004) 074 [hep-th/0312099].

[10] A. Nicolis, R. Rattazzi and E. Trincherini, Phys. Rev. D 79 (2009) 064036 [arXiv:0811.2197 [hep-th]].

[11] E. I. Buchbinder, J. Khoury and B. A. Ovrut, Phys. Rev. D 76 (2007) 123503 [hep-th/0702154].

[12] P. Creminelli, A. Nicolis and E. Trincherini, JCAP 1011 (2010) 021 [arXiv:1007.0027 [hep-th]].

[13] J. Wess and J. Bagger, Princeton, USA: Univ. Pr. (1992) 259 p

[14] J. Khoury, J. -L. Lehners and B. Ovrut, Phys. Rev. D 83 (2011) 125031 [arXiv:1012.3748 [hep-th]].

[15] M. Koehn, J. -L. Lehners and B. A. Ovrut, Phys. Rev. D 86 (2012) 085019 [arXiv:1207.3798 [hep-th]].

[16] F. Farakos and A. Kehagias, JHEP 1211 (2012) 077 [arXiv:1207.4767 [hep-th]].

[17] M. Koehn, J. -L. Lehners and B. A. Ovrut, arXiv:1208.0752 [hep-th].

[18] M. Rocek and A. A. Tseytlin, Phys. Rev. D 59 (1999) 106001 [hep-th/9811232].

[19] S. Sasaki, M. Yamaguchi and D. Yokoyama, Phys. Lett. B 718 (2012) 1 [arXiv:1205.1353 [hep-th]].

[20] M. Kawasaki, M. Yamaguchi and T. Yanagida, Phys. Rev. Lett. 85 (2000) 3572 [hep-ph/0004243].

[21] R. Kallosh, A. Linde and T. Rube, Phys. Rev. D 83 (2011) 043507 [arXiv:1011.5945 [hep-th]].

[22] B. Xue and P. J. Steinhardt, Phys. Rev. Lett. 105 (2010) 261301 [arXiv:1007.2875 [hep-th]].

[23] B. Xue and P. J. Steinhardt, Phys. Rev. D 84 (2011) 083520 [arXiv:1106.1416 [hep-th]].

[24] M. Koehn, J. -L. Lehners and B. Ovrut, arXiv:1212.2185 [hep-th].

[25] J. Khoury, J. -L. Lehners and B. A. Ovrut, Phys. Rev. D 84 (2011) 043521 [arXiv:1103.0003 [hep-th]]. 